\title{
Lesões orofaciais e efeitos colaterais de pacientes HIV positivos em uso da terapia antirretroviral altamente ativa
}

Orofacial lesions and side effects of HIV-positive patients on highly active antiretroviral therapy

Lesiones orofaciales y efectos colaterales de pacientes HIV positivos en uso de la terapia antirretroviral altamente activa

$$
\text { Cristiano Magalhães Moura VILAÇA }{ }^{1}
$$

Michele Pereira de SOUZA ${ }^{2}$

Alan Dornelas SILVA ${ }^{3}$

Katherine Azevedo Batistela Rodigues THULLER $\mathbf{R}^{\mathbf{1}}$

Lívia Gobb LEITE ${ }^{4}$

Ademar TAKAHAMA JÚNIOR ${ }^{5}$

Rebeca de Souza AZEVEDO 6

${ }^{1}$ Programa de Mestrado em Odontologia, Instituto de Saúde de Nova Friburgo, Universidade Federal Fluminense, ISNF - UFF 28625650 Nova Friburgo - RJ, Brasil

\author{
${ }^{2}$ Programa de Pós-Graduação em Ciências Aplicadas a Produtos para a Saúde, Universidade Federal Fluminense, UFF 24020150, Niterói - RJ, Brasil \\ ${ }^{3}$ Pós-Graduação em Odontologia, CETRO, 30140-040 Belo Horizonte - MG, Brasil \\ ${ }^{4}$ Pós-Graduação em Odontologia, Associação Brasileira de Odontologia, ABO, 29160-790 Serra - ES, Brasil \\ ${ }^{5}$ Estomatologia, Universidade Estadual de Londrina, UEL, 86057-970 Londrina - PR, Brasil \\ ${ }^{6}$ Patologia Oral, Instituto de Saúde de Nova Friburgo, Universidade Federal Fluminense, ISNF, UFF, 28625-650 Nova Friburgo - RJ, Brasil
}

\section{Resumo}

Introdução: A AIDS é causada pela infecção do HIV, que progressivamente causa degradação do sistema imunológico do hospedeiro e, consequentemente, o surgimento de diferentes patologias, inclusive na região orofacial. Seu tratamento atua no impedimento da replicação viral, e é chamado de terapia antirretroviral altamente ativa (HAART). Este método terapêutico pode estar associado a alguns efeitos colaterais orofaciais e sistêmicos. Objetivo: Analisar a presença das lesões orofaciais e dos efeitos colaterais associados ao uso da HAART em pacientes HIV+. Material e métodos: Uso de um questionário semiestruturado com informações demográficas, sociais, clínicas e terapêutica, exame físico extra e intraoral, exames laboratoriais e teste de sialometria. Resultados: A amostra foi composta de 43 indivíduos, sendo $53,5 \%$ do sexo feminino com cor de pele branca e idade entre 27 e 58 anos. A maioria refere ter se infectado por meio de atividade sexual (58,1\%) com diagnóstico feito nos últimos 10 anos (68,1\%). A maioria teve carga viral quantificada abaixo do limite mínimo (74,4\%) e contagem de linfócitos T CD4+ acima de 500 células por $\mathrm{mm}^{3}$ de sangue $(56,8 \%)$. Algumas lesões orofaciais, infecções oportunistas e efeitos colaterais ao uso da HAART foram identificados, mas somente a presença de lesões orais foram diretamente relacionadas a baixa quantidade de linfócitos T CD4+ $(p=0,05)$. Conclusão: Todo cirurgião-dentista deve estar atento a presença de lesões orofaciais de pacientes HIV+ porque isso pode indicar uma baixa contagem de linfócitos T CD4+ e, consequentemente, de baixa adesão ou falha na HAART.

Descritores: HIV; Síndrome de Imunodeficiência Adquirida; Terapia Antirretroviral de Alta Atividade.

\section{Abstract}

Introduction: AIDS is caused by HIV infection and progression of events caused in the infected cell, causing the host's immune system degradation and, as a consequence, the development of different pathologies, including orofacial region. Its treatment acts blocking viral replication and is called highly active antiretroviral therapy (HAART). This therapeutic method may be associated with some orofacial and systemic side effects. Objective: To analyze the presence of orofacial lesions and the side effects associated with the use of HAART in HIV+. Material and methods: A semi-structured questionnaire with demographic, social, clinical and therapeutic, extra and intraoral physical exam, laboratorial exams and sialometry. Results: The sample was composed of 43 individuals, in which $53.5 \%$ were female with white skin color and age between 27 and 58 years. Most of them referrer to be infected by means of sexual activity (58.1\%) with a diagnosis made in the last 10 years $(68.1 \%)$. Most of them had viral count under the minimum (74.4\%) and CD4+ lymphocytes above 500 cells per mm3 of blood (56.8\%). Some orofacial lesions, opportunistic infection and side effects from HAART use were identified, but just the presence of oral lesions was directly related with low count of CD4+ lymphocytes $(p=0.005)$. Conclusion: Each dentist should pay attention to orofacial lesions in HIV+ patients because it can indicate a low CD4+ lymphocytes count and, as a consequence, a low adherence or fail in the HAART.

Descriptors: HIV; Acquired Immunodeficiency Syndrome; Antiretroviral Therapy, Highly Active.

\section{Resumen}

Introducción: EI SIDA es causado por la infección del HIV, que progresivamente causa la degradación del sistema inmunológico del huésped y, consecuentemente, el surgimiento de diferentes patologías, incluso en la región orofacial. Su tratamiento actúa en el impedimento de la replicación viral, y se llama terapia antirretroviral altamente activa (HAART). Este método terapéutico puede estar asociado a algunos efectos colaterales orofaciales y sistémicos. Objetivo: Analizar la presencia de las lesiones orofaciales y de los efectos colaterales asociados al uso de la HAART en pacientes HIV+. Material y métodos: Uso de un cuestionario semiestructurado con informaciones demográficas, sociales, clínicas y terapéuticas, examen físico extra e intraoral, exámenes de laboratorio y prueba de sialometría. Resultados: La muestra se compuso de 43 individuos, en los que $53.5 \%$ eran femeninos con color de color blanco y actúan entre 27 y 58 años. En los últimos 10 años (68.1\%), se ha comprobado que se ha infectado por la actividad sexual (58.1\%) con un diagnóstico efectuado en los últimos 10 años (68.1\%). (74.4\%) y CD4+ lymphocytes arriba 500 células per mm3 of blood (56.8\%). Se identificaron algunas lesiones orofaciales, infecciones oportunistas y efectos colaterales al uso de la HAART, pero sólo la presencia de lesiones orales fueron directamente relacionadas con la baja cantidad de linfocitos T CD4+ $(p=0,05)$. Conclusão: Todo cirurgião-dentista deve estar atento a presença de lesões orofaciais de pacientes HIV+ porque isso pode indicar uma baixa contagem de linfócitos T CD4+ e, consequentemente, de baixa adesão ou falha na HAART.

Descriptores: VIH; Síndrome de Inmunodeficiencia Adquirida; Terapia Antirretroviral Altamente Activa.

INTRODUÇÃO

O vírus da imunodeficiência humana (HIV, na sigla em inglês) causa a síndrome da imunodeficiência adquirida (AIDS, na sigla em inglês), doença que, ainda hoje, é um problema de saúde pública mundial ${ }^{1,2}$. Conforme aponta o documento Global AIDS Up Date ${ }^{3}$, em 2017 houveram 1,8 milhão de novas infecções por HIV na população mundial, totalizando 36,9 
milhões de pessoas vivendo com o HIV no mundo.

Após a infecção, o HIV possui afinidade por células responsáveis pela defesa do organismo, os linfócitos TCD4, local no qual o vírus realizará duplicação através da incorporação de seu material genético ao DNA da célula humana com posterior destruição destes linfócitos, e liberação de cópias virais no sistema linfático do hospedeiro, agora capazes de infectar novas células ${ }^{4}$. Estes eventos provocam um declínio na resposta imunológica do hospedeiro, permitindo o aparecimento de infecções oportunistas, ocasionadas principalmente por microrganismos ${ }^{5}$, assim como a progressão da infecção pelo HIV para a AIDS e o desenvolvimento de diferentes outras lesões, inclusive na cavidade oral ${ }^{6}$.

Com o advento da terapia antirretroviral altamente ativa (HAART, na sigla em inglês) ocorreu um aumento significativo do tempo de vida, e consequentemente da qualidade de vida dos indivíduos infectados pelo HIV $\left(\mathrm{HIV}_{+}\right)^{1,7}$, diminuindo a mortalidade e morbidade desta doença, assim como a transmissão do vírus ${ }^{8}$.

No Brasil, em meados de 1992 as medicações antirretrovirais passaram a ser oferecidas, cenário no qual a HAART foi introduzida em novembro de 1996 como parte da política nacional de livre acesso aos serviços de saúde e medicamentos. Assim, a HAART é oferecida para toda a população $\mathrm{HIV}_{+}$com indicação de tratamento ${ }^{1,8}$. De acordo com as informações da página do Ministério da Saúde denominada Infecções Sexualmente Transmitidas - AIDS $^{9}$, os medicamentos antirretrovirais surgiram na década de 1980, para impedir a multiplicação do vírus no organismo e, consequentemente, evitar 0 enfraquecimento do sistema imunológico. Atualmente existem 21 medicamentos disponíveis em 37 apresentações farmacêuticas, sendo divididos em 5 classes: inibidores nucleosídeos da transcriptase reversa (NRTIs, na sigla em inglês); inibidores não nucleosídeos da transcriptase reversa (NNRTIs, na sigla em inglês); inibidores de protease (IP, na sigla em inglês); inibidores de fusão; inibidores de integrase. Na HAART, geralmente são utilizadas pelo menos 3 combinações medicamentosas. Embora esta opção terapêutica tenha trazido uma melhora na qualidade de vida e aumento da sobrevida desta população, alguns efeitos colaterais podem ser vistos $^{5}$ e devem ser levados em consideração durante sua abordagem terapêutica de cada paciente afim de minimizá-los e até impedir uma perda de adesão ao tratamento.
Dessa forma, o objetivo deste trabalho foi analisar as lesões orofaciais e os efeitos colaterais relacionados ao uso da HAART em uma amostra de pacientes $\mathrm{HIV}_{+}$atendidos no município de Nova Friburgo/RJ, ao longo do ano de 2014.

\section{MATERIAL E MÉTODO}

Esta pesquisa foi realizada de acordo com a resolução 196/96 relativa às pesquisas em seres humanos no Brasil, após submissão a aprovação de projeto no Comitê de Ética e Pesquisa em seres Humanos da Universidade Federal Fluminense (CAAE: 28691014.1.0000.5626).

Os pacientes com diagnóstico positivo para a infecção pelo HIV atendidos no Setor de Infectologia na Policlínica da Fundação Municipal de Saúde da cidade de Nova Friburgo foram encaminhados para atendimento na Clínica Odontológica do Instituto de Saúde de Nova Friburgo da Universidade Federal Fluminense de Nova Friburgo (ISNF/UFF). Os pacientes responderam a um questionário semiestruturado contendo informações demográficas, sociais, clínicas e a respeito da infecção pelo HIV, uso da HAART e seus efeitos clínicos. Na sequência foi realizado exame físico extraoral e intraoral para identificação de possíveis lesões.

Foi realizado também teste de sialometria de acordo com o protocolo adotado na clínica de Estomatologia do ISNF/NF. No primeiro teste o paciente, denominado em repouso, com a cabeça levemente inclinada, foi instruído a engolir a primeira saliva e, posteriormente, a cada 5 minutos expelir em um recipiente plástico a saliva que se acumulasse em sua boca. No segundo exame, denominado estimulado, o paciente na mesma posição, foi instruído a mascar uma borracha insípida durante 1 minuto e desprezar a saliva acumulada. Posteriormente, repetir a mesma logística do primeiro exame, porém mascando a borracha. Com o auxílio de uma seringa de 10 $\mathrm{mL}$, as amostras de saliva (com exceção da espuma) dos pacientes produzidas a cada 5 minutos foram mensuradas. Os valores totais de saliva dos testes foram divididos por 5 para se estimar o fluxo salivar em $\mathrm{mL} / \mathrm{min}$ no repouso e no estímulo. Ao final cada paciente foi classificado com hipossalivação, quando os valores de fluxo salivar foram menores que 0,1 $\mathrm{mL} / \mathrm{min}$ e $0,7 \mathrm{~mL} / \mathrm{min}$ para os testes realizados em condição de repouso e em condição de estímulo, respectivamente ${ }^{10,11}$.

Para comparação entre a presença de lesões orofaciais e infecções oportunistas 
relacionadas ao HIV com a taxa de CD4, foi realizado o teste estatístico de Mann-Whitney, considerando $\mathrm{p}<0,05$.

RESULTADOS

Foram incluídos 43 pacientes HIV+ que estavam em tratamento medicamentoso com HAART, dos quais a maioria era do sexo feminino $(53,5 \%)$, cor de pele branca $(53,5 \%)$, com faixa etária variando entre 27 a 58 anos, a maioria estando nas $4^{\underline{a}}$ e $5^{\underline{a}}$ e décadas de vida, e média de idade de 41,6 anos. A maioria destes pacientes contraiu a infecção por relação sexual $(58,1 \%)$ e teve seu diagnóstico nos últimos 10 anos (68,1\%) (Tabela 1).

Tabela 1. Distribuição dos dados sociais e demográficos dos 43 pacientes HIV+ em uso da HAART

\begin{tabular}{|c|c|c|}
\hline Informações Demográficas & Número & $\%$ \\
\hline \multicolumn{3}{|l|}{$\underline{\text { Sexo }}$} \\
\hline Feminino & 23 & 53,5 \\
\hline Masculino & 20 & 46,5 \\
\hline \multicolumn{3}{|l|}{ Faixa etária } \\
\hline $3^{\text {a }}$ década de vida & 3 & 7 \\
\hline $4^{\text {a }}$ década de vida & 14 & 32,6 \\
\hline $5^{\text {a }}$ década de vida & 18 & 41,8 \\
\hline $6^{a}$ década de vida & 8 & 18,6 \\
\hline \multicolumn{3}{|l|}{ Cor de pele } \\
\hline Branca & 22 & 51,2 \\
\hline Parda & 10 & 23,2 \\
\hline Preta & 11 & 25,6 \\
\hline \multicolumn{3}{|l|}{ Forma de contágio } \\
\hline Relação sexual & 25 & 58,1 \\
\hline Transfusão sanguínea & 2 & 4,7 \\
\hline Não souberam informar & 16 & 37,2 \\
\hline \multicolumn{3}{|l|}{ Tempo de diagnóstico } \\
\hline o a 10 anos & 29 & 67,5 \\
\hline 11 a 20 anos & 12 & 27,9 \\
\hline 21 a 30 anos & 1 & 2,3 \\
\hline 31 a 40 anos & 1 & 2,3 \\
\hline
\end{tabular}

A avaliação dos dados sorológicos revelou que a carga viral apresentou resultado abaixo do limite mínimo na maioria dos pacientes $(74,4 \%)$. A contagem de linfócitos $T$ CD4 variou entre 11 e 1509 células/ $\mu$ de sangue, estando predominantemente no intervalo entre 501 a 1000 células (48,8\%). Insta ressaltar que um grupo destes pacientes tinham contagem de linfócitos T CD4 inferior a 500 células $/ \mu$ de sangue $(25,6 \%)$ e, portanto, em manifestação da AIDS. Por fim, a relação CD4/CD8 identificada nestes pacientes variou principalmente entre 0 e 1,5 (55,8\%) (Tabela 2).

Tabela 2. Distribuição dos dados sorológicos dos 43 pacientes HIV+ em uso da HAART

\begin{tabular}{|c|c|c|}
\hline Informações Sorológicas & Número & $\%$ \\
\hline \multicolumn{3}{|l|}{ Carga viral } \\
\hline <limite mínimo & 32 & 74,4 \\
\hline o a 5.000 & 4 & 9,3 \\
\hline Acima de 5.000 & 1 & 2,3 \\
\hline Não quantificada & 6 & 14 \\
\hline \multicolumn{3}{|l|}{ Células CD4+ } \\
\hline o a 500 & 11 & 25,6 \\
\hline 501 a 1000 & 21 & 48,8 \\
\hline 1001 a 1500 & 2 & 4,7 \\
\hline 1501 a 2000 & 1 & 2,3 \\
\hline Não quantificada & 8 & 18,6 \\
\hline \multicolumn{3}{|l|}{ Relação CD4+/CD8+ } \\
\hline o a 0,5 & 9 & 20,8 \\
\hline 0,51 a 1,0 & 13 & 30,3 \\
\hline 1,01 a 1,5 & 2 & 4,7 \\
\hline 1,51 a 2 & 0 & 0 \\
\hline Não quantificada & 19 & 44,2 \\
\hline
\end{tabular}

Em 13 pacientes foram identificadas lesões orais associadas ao HIV $(30,2 \%)$, incluindo candidíase, na forma pseudomembranosa, de queilite angular e eritematosa, leucoplasia pilosa, herpes, do tipo zoster, papiloma e úlcera (Tabela 3) (Figuras 1 e 2). Dentre as infecções oportunistas, quando presente, a pneumonia foi a mais comumente identificada (14 pacientes, 32,6\%) (Tabela 4).

Tabela 3. Lesões orofaciais identificadas nos 43 pacientes HIV+ em uso da HAART*

\begin{tabular}{|c|c|c|}
\hline Lesões Orofaciais & Número & $\%$ \\
\hline Candidíase & 10 & 23,3 \\
\hline Leucoplasia pilosa & 2 & 4,7 \\
\hline Ulcera & 2 & 4,7 \\
\hline Herpes Zoster & 1 & 2,3 \\
\hline Papiloma & 1 & 2,3 \\
\hline $\begin{array}{l}\text { Outras lesões orofaciais não associadas ao } \\
\text { HIV }\end{array}$ & 10 & 23,3 \\
\hline Nenhuma lesão orofacial associada ao HIV & 25 & 58,1 \\
\hline
\end{tabular}

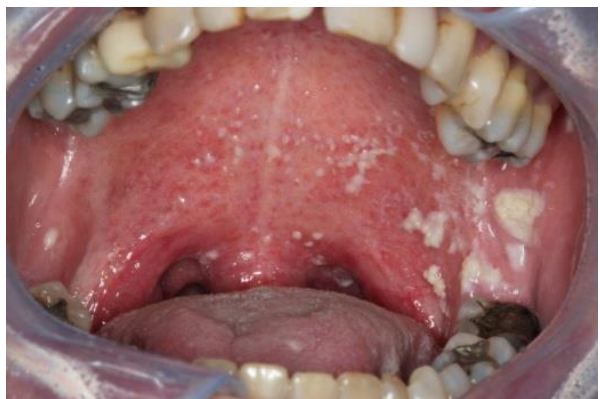

Figura 1: Fotografia clínica evidenciando placas brancas destacáveis em mucosa jugal, palato duro e mole, úvula e trígono retromolar, compatível com o diagnóstico de candidíase pseudomembranosa.

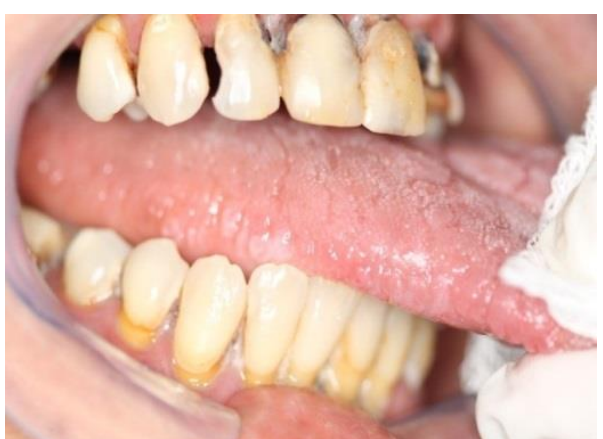

Figura 2: Fotografia clínica evidenciando placa branca com superfície pregueada e bordas irregulares em borda lateral de língua, compatível com o diagnóstico de leucoplasia pilosa oral.

Tabela 4. Infecções oportunistas identificadas nos 43 pacientes HIV+ em uso da HAART

\begin{tabular}{|lcc|}
\hline \multicolumn{1}{|c}{ Infecções oportunistas } & Número & $\%$ \\
\hline Pneumonia & 14 & 32,6 \\
\hline Tuberculose & 7 & 16,3 \\
\hline Herpes Zoster & 4 & 9,3 \\
\hline Candidíase & 4 & 9,3 \\
\hline Sarcoma de Kaposi & 1 & 2,3 \\
\hline Nenhuma infecção oportunista & 13 & 30,2 \\
\hline
\end{tabular}

Os medicamentos mais utilizados na HAART desta amostra de pacientes foram: Kaletra ${ }^{\circledR}$ (lopinavir e ritonavir) do grupo dos inibidores de proteases (11 pacientes, 25,6\%), Biovir ${ }^{\circledR} /$ Lafepe ${ }^{\circledR}$ (lamivudina + zidovudina) do grupo dos inibidores nucleosídeos da transcriptase reversa (24 pacientes, 55,8\%),

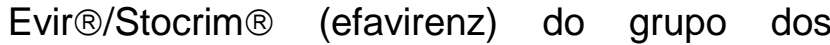
inibidores não nucleosídeos da transcriptase 
reversa (18 pacientes, 41,8\%), geralmente em associação uns com os outros dentro do seu esquema terapêutico individual da HAART. Oito pacientes não souberam informar quais medicamentos estavam sendo utilizados na sua HAART (Tabela 5).

Tabela 5. Distribuição dos medicamentos em uso pelos 43 pacientes HIV+ em uso da HAART

\begin{tabular}{|c|c|c|}
\hline Medicamentos & Número & $\%$ \\
\hline \multicolumn{3}{|l|}{ IP } \\
\hline Kaletra® & 11 & 25 \\
\hline Ritonavir & 7 & 15,9 \\
\hline Atazanavir & 5 & 11,4 \\
\hline Darunavir & 1 & 2,3 \\
\hline \multicolumn{3}{|l|}{ NRTI } \\
\hline Biovir $\AA /$ Lafepe $®$ & 24 & 54,5 \\
\hline Lamivudina & 11 & 25 \\
\hline Tenofovir & 4 & 9,1 \\
\hline Estavudina & 1 & 2,3 \\
\hline Abacavir & 1 & 2,3 \\
\hline \multicolumn{3}{|l|}{ NNRTI } \\
\hline Efavirenz/Stocrim $\mathbb{}$ & 18 & 40,9 \\
\hline \multicolumn{3}{|l|}{ Inibidores de fusão } \\
\hline Enfuvirtida & 1 & 2,3 \\
\hline \multicolumn{3}{|l|}{ Inibidores de integrase } \\
\hline Raltegravir & 1 & 2,3 \\
\hline Não informaram & 6 & 14 \\
\hline
\end{tabular}
NNRTI = inibidores não nucleosídeos da transcriptase reversa.

Os efeitos colaterais mais frequentes, relatados pelos pacientes foram náuseas $(10$ pacientes, 23,3\%), tontura (8 pacientes, 18,6\%), xerostomia (5 pacientes, $11,6 \%$ ) e diarreia (5 pacientes, 11,6\%) (Tabela 6).

Tabela 6. Principais efeitos colaterais descritos pelos 43 pacientes HIV+ em uso da HAART.

\begin{tabular}{|lcc|}
\hline \multicolumn{1}{|c}{ Efeitos colaterais } & Número & $\%$ \\
\hline Náusea & $\mathbf{1 0}$ & $\mathbf{2 3 , 3}$ \\
\hline Tontura & 8 & $\mathbf{1 8 , 6}$ \\
\hline Xerostomia & 5 & $\mathbf{1 1 , 6}$ \\
\hline Diarreia & 5 & $\mathbf{1 1 , 6}$ \\
\hline Alteração do paladar & 4 & 9,3 \\
\hline Alterações psicológicas & 3 & 7 \\
\hline Nenhum efeito colateral & $\mathbf{8}$ & $\mathbf{1 8 , 6}$ \\
\hline
\end{tabular}

O teste de sialometria foi realizado em 12 pacientes, os resultados demonstraram que 10 pacientes $(83,3 \%)$ apresentaram fluxo salivar normal e 2 pacientes (16,7\%) hipossalivação.

A análise estatística revelou uma associação significativa entre a presença de lesões orais associadas ao HIV e a menor contagem de linfócitos T CD4 $(p=0,05)$ (Figura $3)$, mas não revelou a mesma associação entre a presença de infecções oportunistas e a contagem maior ou menor de linfócitos T CD4 $(p=0,9)$.

\section{DISCUSSÃO}

$\mathrm{Na}$ evolução do HIV+ para a AIDS observa-se um aumento da contagem da carga viral e uma redução da contagem de linfócitos $T$ CD4, que, desta forma, levará a uma diminuição da atuação do sistema de defesa, justificando o aparecimento de diferentes lesões, incluindo infecções oportunistas, em especial as associadas a microrganismos ${ }^{5}$ como a candidíase oral e herpes.

A contagem de linfócitos T CD4+ abaixo de 500 células $/ \mathrm{mm}^{3}$ é um importante indicativo para o início da HAART, por compreender que neste momento estes pacientes detenham de um sistema imunológico altamente debilitado ${ }^{5}$. Cerca de $75 \%$ dos pacientes deste estudo apresentaram exames com contagem de linfócitos T CD4+ acima deste valor referência no dia da primeira consulta, que, junto com a carga viral indetectável também identificada na maioria destes pacientes, indica que estes pacientes, mesmo portadores do HIV, estejam em um bom estado de saúde e, assim, possivelmente fora de risco de novas infecções ${ }^{8,12}$.

Contudo, cerca de um terço destes pacientes possuía baixos valores de linfócitos TCD4+, mesmo em adesão terapêutica, percentual semelhante e previamente identificado em uma amostra de pacientes vivendo com HIV/AIDS na Ásia ${ }^{13}$. Com isso, é possível afirmar que os profissionais que lidam com este perfil de paciente precisam manter um acompanhamento e atenção especiais, visto que eles podem exibir patologias associadas a doença e piora progressiva do quadro ${ }^{14}$. Neste contexto, é importante ressaltar que a baixa de contagem de linfócitos TCD4+ estava associada de forma significante ao desenvolvimento de lesões orofaciais, assim como as condições de saúde oral como um todo, incluindo o desenvolvimento de cárie e doença periodontal ${ }^{15}$. Tal associação pode servir de indicativo para que a avaliação dos exames laboratoriais de contagem de linfócitos TCD4+ em conjunto com um detalhado exame físico e conhecimento das lesões orofaciais associadas ao $\mathrm{HIV}+$ sejam procedimentos essenciais que 0 cirurgião-dentista deve ter no manejo destes pacientes. Adicionalmente, é também essencial que este cirurgião-dentista se mantenha em íntimo contato com a equipe de infectologia deste paciente, informando sobre o aparecimento ou não de lesões orofaciais, que podem auxiliar na identificação de uso irregular ou falha da terapêutica empregada.

A maioria dos pacientes $\mathrm{HIV}_{+}$desta amostra de estudo apresentavam alguma infecção oportunista, que por muitas vezes quando presentes auxiliam no manejo e diagnóstico da AIDS ainda não identificada, como a candidíase, seja ela oral ou não ${ }^{5}$. Tal constatação é interessante porque, ainda que os exames laboratoriais estejam dentro do esperado e os pacientes refiram o uso regular da HAART, estas infecções seguem ocorrendo. Neste caso, poderíamos esperar que, assim como identificado nas lesões orofaciais, este achado esteja diretamente relacionado à baixa 
contagem de linfócitos TCD4+, mas, não. Ao menos nesta amostra de pacientes, não foi identificada esta correlação e as infecções oportunistas ocorreu de forma semelhante independente da presença de maior ou menor quantidade de linfócitos TCD4+.

Herpes simples, candidíase, leucoplasia pilosa e sarcoma de Kaposi são lesões associadas a diferentes microrganismos que podem estar diretamente associadas ao processo HIV/AIDS, podendo, inclusive, muitas vezes, servir de fonte inicial para o diagnóstico da doença nestes pacientes ${ }^{14,16-19}$.

A HAART tem fundamental importância e papel na vida destes pacientes de forma que permitiu um aumento significativo na sua qualidade de vida e também na sobrevida ${ }^{7}$, o que pode ser bem exemplificado em nossa amostra, quando cerca de $1 / 3$ de todos os pacientes estejam vivendo com a doença há mais de 10 anos, um deles vivendo entre 30 e 40 anos com a doença controlada e em bom estado de saúde, o que em época anterior à sua implementação não poderia acontecer tão facilmente. E, por isso, com a HAART tendo como principal foco 0 impedimento da replicação viral e consequente manutenção de valores viáveis de linfócitos TCD4+, o tratamento permite esta conquista em longo prazo. Todavia, em paralelo a todas estas vantagens, pode provocar uma mudança no perfil das lesões orais e o surgimento de efeitos colaterais associados com a medicação diária, que são ministradas em doses elevadas e de forma multimedicamentosa.

Desde a implementação da HAART, as alterações bucais mais comumente observadas nos pacientes HIV positivos com AIDS estão relacionadas ao papiloma vírus humano (HPV), ao vírus do herpes simples (HSV) e ao vírus varicela zoster $(\mathrm{VZV})^{4}$, algumas delas também identificadas nesta amostra de pacientes HIV+. Seguindo esta linha em associação a HAART, alguns efeitos colaterais podem ser observados, como náuseas, tontura, xerostomia e diarreia, identificados neste estudo, e que podem reduzir a adesão dos pacientes ao uso regular e ininterrupto da HAART, e consequentemente, a sua eficácia no controle da AIDS $^{20}$. Dentre os efeitos colaterais diretamente relacionados a odontologia, destaca-se a xerostomia e a disgeusia, que representam, respectivamente a sensação de boca seca e alteração do paladar, que podem estar associadas a redução efetiva do fluxo salivar, e provavelmente associada a ministração da medicação individual. Tais queixas estiveram exclusivamente presentes em pacientes que utilizaram Biovir ${ }^{\circledR}$, do grupo dos inibidores nucleosídeos da transcriptase reversa, e Evir ${ }^{\circ} /$ Stocrim $\AA$, do grupo dos inibidores não nucleosídeos da transcriptase reversa, que em uma revisão sistemática da literatura recente foram os grupos de medicamentos mais associados a efeitos colaterais bioquímicos ${ }^{21}$.

CONCLUSÃO

O grupo de pacientes $\mathrm{HIV}_{+}$em uso de principalmente de mulheres brancas e adultas, que contraiu a infecção por relação sexual sem proteção e teve seu diagnóstico nos últimos 10 anos. A maioria destes pacientes está bem controlada e exibe carga viral e contagem de linfócitos $\mathrm{T}$ CD4+ dentro da normalidade, contudo, ainda assim, foram observadas infecções oportunistas em lesões orofaciais, estas diretamente relacionadas com a menor quantidade de linfócitos T CD4+ circulantes no sangue, e que devem ser sempre avaliados pelo cirurgião-dentista que atende este grupo de pacientes.

Efeitos colaterais foram relatados pela maioria dos pacientes e devem também ser levados em consideração pelo cirurgião-dentista em acompanhamento destes pacientes, pelo fato de que estes podem reduzir a adesão a HAART e, consequentemente, perda de controle da AIDS e piora progressiva do quadro.

\section{REFERÊNCIAS}

1. Geogze M, Mucci S, Marco MA, Martins LAN, Citeripo VA. Qualidade de vida e Adesão ao tratamento anti-retroviral de pacientes portadores de HIV. Rev Saúde Pública. 2010; 44:743-49.

2. Nungu SI, Mghamba JM, Rumisha SF, Semali IA. Uptake and determinants for HIV postpartum re-testing among mothers with prenatal negative status in Njombe region, Tanzania. BMC Infect Dis. 2019;19(1):398.

3. Global AIDS up date 2019, disponível em: https://www.unaids.org/es

4. Costa DCB, Sarmento DJS, Silveira EJD. Manifestações orais em pacientes HIV + na era da terapia antiretroviral de alta atividade: o que mudou? - uma atualização para o clínico. Int $\mathrm{J}$ Dent. 2011; 10(2): 97-102.

5. Patton LL. Oral lesions associated with human immunodeficiency virus disease. Dent Clin North Am. 2013;57(4):673-98.

6. Palella FJ Jr, Delaney KM, Moorman AC, Loveless MO, Fuhrer J, Satten GA, Aschman DJ, Holmberg SD. Declining morbidity and mortality among patients with advanced human immunodeficiency virus infection. HIV Outpatient Study Investigators. N Engl J Med. 1998;338(13):853-60. 
7. Eliozondo JE, Trevino AC, Violant D. Dentistry and HIV/AIDS related stigma. Rev Saúde Pública. 2015;49:79.

8. Pacheco PRG, Zara ALSA, Silva E Souza LC, Turchi MD. Late Onset of Antiretroviral Therapy in Adults Living with HIV in an Urban Area in Brazil: Prevalence and Risk Factors. J Trop Med. 2019;2019:5165313.

9. Departamento de IST, AIDS e hepatites virais. Quais são os antirretrovirais. Disponível em: www.aids.gov.br/pagina/quais-sao-osantirretrovirais.

10. Barbe AG, Ludwar L, Hamacher S, Noack MJ. Efficacy of a newly developed mouth gel for xerostomia relief-A randomized double-blind trial. Oral Dis. 2019;25(6):1519-29.

11. Lopez-Pintor RM, Casañas E, GonzalesSerrano J, Serrano J, Ramirez L, de Arriba L, et al. Xerostomia, Hypossalivation, and Salivary Flow in Diabetes Patients. J Diabetes Res. 2016. doi:10.1155/2016/4372852.

12. Margalho R, Pereira M, Ouakinin S, Canavarro MC. Adesão à HAART, qualidade de vida e sintomat ologia psicopat ológica em doentes infectados pelo $\mathrm{VIH} / \mathrm{SIDA}$ [Adherence to HAART, quality of life and psychopathological symptoms among HIV/AIDS infected patients]. Acta Med Port. 2011;24(Suppl 2):539-48.

13. Mwangosi IE, Tillya J. Oral lesions associated with HIV/AIDS in HIV-seropositive patients attending a counselling and treatment centre in Dar es Salaam. Int Dent J. 2012;62(4):197-202.

14. Patton LL, Ramirez-Amador V, AnayaSaavedra G, Nittayananta W, Carrozzo M, Ranganathan K. Urban legends series: oral manifestations of HIV infection. Oral Dis. 2013;19(6):533-50.

15. Souza AJ, Gomes-Filho IS, Silva CALD, Passos-Soares JS, Cruz SSD, Trindade SC et al. Factors associated with dental caries, periodontitis and intra-oral lesions in individuals with HIV / AIDS. AIDS Care. 2018;30(5): 578-85.

16. Ablanedo-Terrazas $Y$, Alvarado-de la Barrera C, Ormsby CE, Reyes-Terán G. Head and neck manifestations of the immune reconstitution syndrome in HIV-infected pat5ents: a cohort study.Otolaryngol Head Neck Surg. 2012;147(1): 52-6.

17. Cepeda LAG, Salobrena AC, Ortega KL, Mora NA, Soriano YJ. Oral lesions and immune reconstitution syndrome in HIV+/AIDS patient receiving highly active antiretroviral therapy. Epidemiological evidence. Med Oral Patol Oral Cir Bucal. 2008; 13(2): 85-93.

18. Papagatsia Z, Jones J, Morgan P, Tappuni AR. Oral Kaposi sarcoma: a case of immune reconstitution inflammatory syndrome. Oral Surg Oral Med Oral Pathol Oral Radiol Endod. 2009;108(1):70-5.
19. Ramírez-Amador VA, Espinosa E, GonzálezRamírez I, Anaya-Saavedra G, Ormsby CE, Reyes-Terán G. Identification of oral candidosis, hairy leukoplakia and recurrent oral ulcers as distinct cases of immune reconstitution inflammatory syndrome. Int $\mathrm{J}$ STD AIDS. 2009;20(4):259-61.

20.Zhang L, Li X, Lin Z, Jacques-Tiura AJ, Xu J, Zhou $Y$ et al. Side effects, adherence selfefficacy, and adherence to antiretroviral treatment: a mediation analysis in a Chinese sample. AIDS Care. 2016;28(7):919-26.

21. Silva BF, Peixoto G, da Luz SR, de Moraes S, Peres SB. Adverse effects of chronic treatment with the Main subclasses of highly active antiretroviral therapy: a systematic review. HIV Med. 2019;20(7):429-38.

\section{CONFLITO DE INTERESSES}

Os autores declaram não haver conflitos de interesse

AUTOR PARA CORRESPONDÊNCIA

\section{Ademar Takahama Júnior}

Estomatologia. Universidade Estadual de Londrina.

Rodovia Celso Garcia Cid - PR 445 Km 380

Campus Universitário - Cx. Postal 10.011

86.057-970 - Londrina-PR, Brasil

Telefone: 4333716723

E-mail: ademartjr@uel.br
Submetido em 20/05/2019

Aceito em 28/08/2020 